\title{
Polaronic orbital polarization in a layered colossal magnetoresistive manganite
}

\author{
B. J. Campbell, ${ }^{1, *}$ S. K. Sinha, ${ }^{2,3}$ R. Osborn, ${ }^{1}$ S. Rosenkranz, ${ }^{1}$ J. F. Mitchell, ${ }^{1}$ D. N. Argyriou,,${ }^{1}$ L. Vasiliu-Doloc, ${ }^{4}$ \\ O. H. Seeck, ${ }^{4}$ and J. W. Lynn ${ }^{5}$ \\ ${ }^{1}$ Materials Science Division, Argonne National Laboratory, Argonne, Illinois 60439 \\ ${ }^{2}$ Department of Physics, University of California at San Diego, La Jolla, California 92093 \\ ${ }^{3}$ Los Alamos Neutron Science Center, Los Alamos National Laboratory, Los Alamos, New Mexico 87545 \\ ${ }^{4}$ Advanced Photon Source, Argonne National Laboratory, Argonne, Illinois 60439 \\ ${ }^{5}$ NIST Center for Neutron Research, National Institute of Standards and Technology, Gaithersburg, Maryland 20899
}

(Received 30 August 2002; published 31 January 2003)

\begin{abstract}
The striking anisotropy observed in Huang scattering distributions near the intense Bragg reflections of $\mathrm{La}_{1.2} \mathrm{Sr}_{1.8} \mathrm{Mn}_{2} \mathrm{O}_{7}$ is shown to be the result of orbitally polarized polarons in the paramagnetic insulating state above $T_{C}$. X-ray single-crystal diffuse scattering patterns from this bilayered colossal magnetoresistive manganite are calculated in terms of the polaronic local structure and compared with experimental measurements. At $300 \mathrm{~K}$, the polaronic $e_{g}$ electrons occupy "out-of-plane" (i.e., $3 z^{2}-r^{2}$ ) orbitals, leading to $\mathrm{MnO}_{6}$ octahedra that are Jahn-Teller elongated along the $c$ axis, perpendicular to the perovskite layers. Between $300 \mathrm{~K}$ and $T_{C}$, however, the "orbital polarization" is shown to shift into the $\boldsymbol{a}$ - $\boldsymbol{b}$ plane (i.e., $3 x^{2}-r^{2}$ and $3 y^{2}-r^{2}$ orbitals), allowing the formation of nanoscale polaron correlations above $T_{C}$.
\end{abstract}

DOI: 10.1103/PhysRevB.67.020409

The Jahn-Teller (JT) distortions associated with polaronic charge carriers in the colossal magnetoresistive manganites are one of the most important physical expressions of colossal magnetoresistance (CMR) phenomena, providing a unique structural probe of the competing interactions that govern their complex electronic, magnetic, and structural phase diagrams. ${ }^{1}$ These subtle structures can be studied in detail via advanced $\mathrm{x}$-ray and neutron-scattering tools as functions of physical properties, external fields, and synthetic processing variables. Incoherent local distortions, for example, probe the behavior of JT polarons ${ }^{2-5}$ through the unusual paramagnetic insulator (PI) to ferromagnetic metal (FM) transition, while coherent JT-coupled superstructures probe the development of long-range charge and orbitallyordered insulating states. ${ }^{6-9}$ There has been considerable recent interest in short-range polaronic correlations ${ }^{2,10-14}$ that track the rise and fall of the resistivity across the PI-FM transition. The formation of these features is not well understood, particularly in the layered perovskites, where longitudinal and transverse JT correlations coexist and possibly compete. ${ }^{14}$ In the present work, we report a form of polaronic order in $\mathrm{La}_{1.2} \mathrm{Sr}_{1.8} \mathrm{Mn}_{2} \mathrm{O}_{7}$ that facilitates the formation of nanoscale polaron correlations.

In $\mathrm{La}_{1-x} \mathrm{Ca}_{x} \mathrm{MnO}_{3}$, powder pair distribution function (PDF) and $\mathrm{x}$-ray absorption fine structure (XAFS) analyses have been employed to identify the presence of hole-type $\mathrm{Mn}$ $d\left(e_{g}\right)$ polarons in the paramagnetic insulating state that disappear below $T_{C}$, and to obtain an isotropic measure of the local $\mathrm{Mn}-\mathrm{O}$ distortions. ${ }^{3,4}$ Neutron powder diffraction data from $\mathrm{La}_{2-2 x} \mathrm{Sr}_{1+2 x} \mathrm{Mn}_{2} \mathrm{O}_{7}$ reveal similarly anomalous increases in the Debye-Waller factors just above $T_{C}$, and demonstrate that these phenomena extend to the layered perovskite systems. ${ }^{5}$ While techniques that involve powder samples have been extensively developed, so that data collection is relatively straightforward, the analysis of diffuse scattering from powder data does lead to directionally averaged information. Single-crystal diffuse scattering methods, on the other hand, probe polaronic local structure in three- dimensional (3D) detail, without the severe feature-overlap problems incurred when collapsing 3D reciprocal space features down to one dimension. The recent x-ray diffuse scattering study by Campbell et al. ${ }^{14}$ for example, yielded a detailed 3D model of polaronic correlation in $\mathrm{La}_{1.2} \mathrm{Sr}_{1.8} \mathrm{Mn}_{2} \mathrm{O}_{7}$ that exhibited length scales between 10 and $30 \AA$.

Striking single-crystal diffuse scattering patterns have been reported around the Bragg reflections of $\mathrm{La}_{1.2} \mathrm{Sr}_{1.8} \mathrm{Mn}_{2} \mathrm{O}_{7}$, and are attributed to polaronic Jahn-Teller distortions in the paramagnetic insulating state above $T_{C}{ }^{2}$ Quasielastic neutron-scattering measurements reveal these highly anisotropic features to be static on the 10-ps time scale. Their intensity falls off like $1 / q^{2}$ away from the Bragg scattering vector, indicating that local polaronic distortions behave like anisotropic point defects in an elastic medium, as illustrated in Fig. 1. Known as Huang scattering, ${ }^{15}$ such a distribution is not produced directly from the local displacements at the core of the JT distortion, but rather from the long-range strain field that arises in the crystalline lattice in response to the local displacements. Due to the inverse rela-

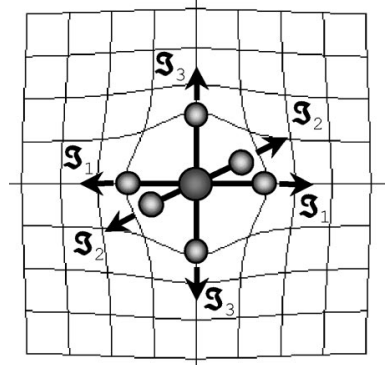

FIG. 1. Illustration of the model of an embedded $\mathrm{MnO}_{6}$ octahedron in an elastic continuum matrix. The applied local forces $\mathfrak{I}_{n}$ at the positions of the apical oxygens are balanced by the induced stresses in the matrix. The long-range strain field that develops, illustrated by the warped grid lines, gives rise to the butterflyshaped Huang scattering patterns observed in $\mathrm{x}$-ray diffuse scattering data. 
tionship between real and reciprocal space features, the scattering near the Bragg peak arises from the long-range strain, while the polaronic local displacements produce scattering that extends far from the Bragg peaks. The resulting scattering distribution can be simply interpreted as the magnitude of the Fourier transform of the long-range strain field. Obviously, the scattering near the Bragg peak is more easily handled because it is fairly localized in reciprocal space. The direct scattering from the local displacements is widely distributed, thereby making it weaker and more difficult to measure and analyze.

The local displacements can still be probed, however, by force-coupling them to the long-range displacements using the method of static displacement waves. ${ }^{16}$ Because the measurable Huang scattering distribution is close to the Bragg peak, where the acoustic phonon frequencies are the dominant contributors, we can conveniently treat the dynamical matrix in the continuum elastic approximation, which only requires replacing the eigenvectors and frequencies of the lattice dynamical matrix with those of the elastic dynamical matrix, and eliminating the optic phonons. Conceptually, we (a) treat the crystal as a continuous elastic medium, (b) embed an $\mathrm{MnO}_{6}$ octahedron in the medium, (c) apply balanced forces to the apical oxygens, (d) allow the medium to respond to this local distortion by forming a long-range strain field, and then (e) calculate the diffuse scattering due to this strain field.

In this approximation, the diffuse polaron scattering ${ }^{17}$ can be expressed as

$$
\begin{aligned}
I_{\mathrm{POL}}(\mathbf{Q})= & N\left|F_{\mathbf{G}}\right|^{2} x(1-x) \sum_{\alpha, \beta, \gamma, \delta} Q_{\beta} Q_{\delta} \\
& \times\left(\sum_{j, j^{\prime}} \frac{\varepsilon_{\alpha, \mathbf{q}, j} \varepsilon_{\beta, \mathbf{q}, j}^{*} \varepsilon_{\gamma, \mathbf{q}, j^{\prime}}^{*} \varepsilon_{\delta, \mathbf{q}, j^{\prime}}}{M^{2} \omega_{\mathbf{q}, j^{\prime}}^{2} \omega_{\mathbf{q}, j^{\prime}}^{2}}\right) \\
& \times \sum_{m, n} \mathfrak{I}_{m, \alpha} \mathfrak{I}_{n, \gamma} e^{i \mathbf{q} \cdot\left(\mathbf{R}_{m}-\mathbf{R}_{n}\right)}
\end{aligned}
$$

where $N$ is the number of unit cells in the crystal, $M(1092.6$ $\mathrm{g} / \mathrm{mol}$ ) is the unit cell mass, $x$ is the doping level, $F_{\mathbf{G}}$ is the structure factor for the Bragg reflection $\mathbf{G}, \varepsilon_{\alpha, \mathbf{q}, j}$ and $\omega_{\mathbf{q}, j}$ are the respective eigenvectors and eigenfrequencies of the continuum elastic dynamical matrix associated with position $\mathbf{q}$ $=\mathbf{Q}-\mathbf{G}$ and phonon branch $j, \mathfrak{I}_{m, \beta}$ is the Kanzaki force ${ }^{18}$ applied by a local JT distortion to local atom $m$ located at $\mathbf{R}_{m}$, and greek indices represent vector components. The scattering distribution surrounding reciprocal lattice vector $\mathbf{G}$ also includes a contribution from thermal diffuse scattering (TDS), ${ }^{2}$ which can similarly be expressed as

$I_{\mathrm{TDS}}(\mathbf{Q})=N\left|F_{\mathbf{G}}\right|^{2}\left(\frac{k T}{2}\right) \sum_{\beta, \delta} Q_{\beta} Q_{\delta}\left(\sum_{j} \frac{\varepsilon_{\beta, \mathbf{q}, j}^{*} \varepsilon_{\delta, \mathbf{q}, j}}{M \omega_{\mathbf{q}, j}^{2}}\right)$,

where $k T$ is the thermal energy scale. The TDS is only weakly anisotropic compared to the polaron scattering, but comparable in intensity. We have found that it is sufficient in the present situation to include Kanzaki forces only on the six oxygens of a single Jahn-Teller-distorted $\mathrm{MnO}_{6}$ octahe- dron. This involves three balanced central forces $\left(\mathfrak{I}_{\mathrm{O} 1, x}, \mathfrak{I}_{\mathrm{O} 2, y}, \mathfrak{I}_{\mathrm{O} 3, z}\right)$ as shown in Fig. 1. Quantitative local structural detail is extracted by treating the unknown forces as fitting parameters, while comparing the calculated (i.e., $\left.I_{\mathrm{POL}}+I_{\mathrm{TDS}}\right)$ and experimental diffuse scattering patterns.

The collection of 36-keV x-ray diffuse scattering data from a $6 \times 4 \times 1-\mathrm{mm}^{3}$ single-crystal sample of $\mathrm{La}_{1.2} \mathrm{Sr}_{1.8} \mathrm{Mn}_{2} \mathrm{O}_{7}$ at the SRI 1-ID-C beamline at the Advanced Photon Source was described previously. ${ }^{2}$ Lowtemperature measurements employed a He displex unit and $\mathrm{x}$-ray detection employed a Ge solid-state detector. Neutron triple-axis measurements of six independent accoustic phonon velocities were conducted using the BT-2 and BT-9 spectrometers at the NIST research reactor, with a graphite monochromator, filter, and analyzer. These velocities were used to construct the elastic dynamical matrix. Crystallographic indices refer to a standard unit cell with $I 4 / \mathrm{mmm}$ symmetry and lattice parameters: $\boldsymbol{a} \sim 3.86 \AA$ and $\boldsymbol{c}$ $\sim 20.05 \AA$.

A two-dimensional fit of the calculated diffuse scattering in the $(h 0 l)$ section surrounding the $(200)$ Bragg reflection against experimental $\mathrm{x}$-ray single-crystal diffuse scattering patterns has been employed to extract the three force parameters of this model at $T=300 \mathrm{~K}$. The calculated and experimental results are shown as absorption-corrected log-scale contour plots in Fig. 2(a), including a panel that adds artificial noise to the calculated pattern in order to provide a more realistic comparison. The vector displacement of the atom at $\mathbf{R}_{m}$, defined as its local position minus its average position (i.e., $\mathbf{u}_{m}=\mathbf{r}_{m}-\mathbf{R}_{m}$ ) can be expressed in terms of the local forces as

$$
\begin{aligned}
u_{m, \alpha}= & \int \frac{d^{3} q}{(\pi / a)^{3}} \sum_{\beta}\left(\sum_{j} \frac{\varepsilon_{\beta, \mathbf{q}, j}^{*} \varepsilon_{\alpha, \mathbf{q}, j}}{M \omega_{\mathbf{q}, j}^{2}}\right) \\
& \times\left(\sum_{n} \Im_{n, \beta} e^{i \mathbf{q} \cdot\left(\mathbf{R}_{m}-\mathbf{R}_{n}\right)}\right),
\end{aligned}
$$

which involves a $q$-space integral over a sphere of radius $2 \pi / a$. Once determined, the local forces are inserted into this expression, and the $q$-space integration is carried out numerically. The local oxygen displacements determined in this fashion are quantitative on an absolute scale by virtue of the fact that the TDS contribution to the diffuse scattering pattern serves as a built-in reference against which the strength of the polaronic contribution is measured. The TDS contribution is completely determined by the dynamical matrix and the temperature.

At room temperature and $\mathbf{G}=\left(\begin{array}{lll}2 & 0 & 0\end{array}\right)$, the threedimensional Jahn-Teller distortion was found to consist of local displacements $u_{\mathrm{O} 1, x}=u_{\mathrm{O} 2, y}=-0.012(3) \AA$ and $u_{\mathrm{O} 3, z}$ $=0.074(8) \AA$. By symmetry, each vector displacement includes one nonzero component parallel to the corresponding force. The $u_{\mathrm{O} 1, x}$ and $u_{\mathrm{O} 2, y}$ displacements were set equal because the fit only tolerated a small difference between the two that was found to be negligible. This effectively leaves two free parameters in the local distortion: an overall displacement scale factor and an out-of-plane to in-plane displacement ratio. Both of these parameters strongly influence the depths and curvatures of the cusps and the overall shape 

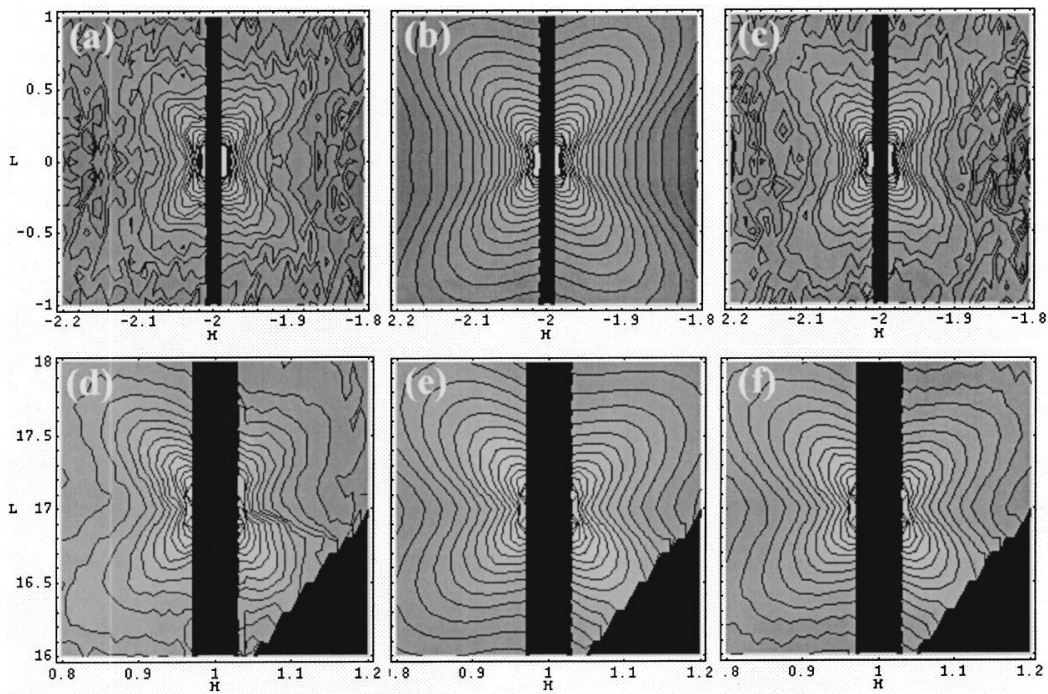

FIG. 2. Calculated and experimental x-ray (36 $\mathrm{keV})$ diffuse scattering data from the $(h 0 l) \mathrm{sec}-$ tions surrounding the $\left(\begin{array}{lll}2 & 0 & 0\end{array}\right)$ Bragg reflection [panels (a)-(c)] and the (1 0 17) Bragg reflection [panels (d)-(f)] at room temperature. Panels (a) and (d) contain experimental patterns, whereas panels (b) and (c) contain calculated patterns. Panels (c) and (f) are identical to (b) and (e) except that artificial noise has been added at the experimentally observed noise level in order to provide a more realistic comparison. of the pattern. The overall magnitude of the local JT distortions observed here is roughly half that of the coherent JT distortion observed in orbitally-ordered $\mathrm{La}_{0.5} \mathrm{Ca}_{0.5} \mathrm{MnO}_{3},{ }^{6}$ but nevertheless significant.

We must consider the fact that $e_{g}$ electrons have both $d\left(3 z^{2}-r^{2}\right)$ and $d\left(x^{2}-y^{2}\right)$ type orbitals available, each of which can be oriented in three different ways, i.e., with the symmetry axis parallel to $\boldsymbol{a}, \boldsymbol{b}$, or $\boldsymbol{c}$. One can imagine a purely random orbital configuration that allows each of the three orbital orientations to occur with equal probability so as to yield a relatively isotropic diffuse scattering pattern. In contrast, the strong anisotropy of the observed butterflyshaped pattern and the local distortion that produces it reveal a population of Jahn-Teller polarons that are orbitally polarized. The large octahedral stretch parallel to the $c$ axis is accompanied by a much smaller symmetric compression in the $\boldsymbol{a} \boldsymbol{-} \boldsymbol{b}$ plane, indicating that the polarons primarily occupy $d\left(3 z^{2}-r^{2}\right)$ orbitals. This effect is not the same as orbital ordering, as occurs in $\mathrm{LaMnO}_{3}$. In the present case, the polaronic orbitals responsible for the Huang scattering are orientationally correlated, but not spatially correlated. Evidence of $d\left(x^{2}-y^{2}\right)$ orbital polarization has previously been reported for the FM state below $T_{C},{ }^{19-21}$ but the observation of polaronic orbital polarization in the PI state above $T_{C}$ was not made earlier.

The $(h 0 l)$ sections shown in Fig. 2 contain fully threedimensional structural information due to the fact that the elastic constants couple a force directed along one axis to the displacements along the other two. This section was chosen because it is adequately sensitive to each of the three force parameters. In contrast, the $\left(\begin{array}{lll}0 & 0 & l\end{array}\right)$ section is less useful, because the superposition of local distortion anisotropies along the two symmetry-related $\boldsymbol{a}^{*}$ and $\boldsymbol{b}^{*}$ directions yields a relatively isotropic result. Superposition is still accounted for in the ( $h 0 l)$ sections, but does not wash out the anisotropy. It is accommodated by computing each pattern twice, with local distortions that are related by a $90^{\circ} \boldsymbol{a}-\boldsymbol{b}$ plane rotation, and averaging the results. Note that the same set of local displacements that matched the $\left(\begin{array}{lll}2 & 0 & 0\end{array}\right)$ diffuse scattering pattern also reproduces the diffuse scattering around the

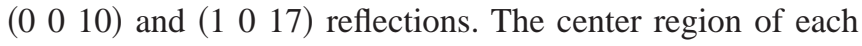
pattern in Fig. 2 has been removed in order to eliminate the layered-intergrowth defect rod, which is temperature independent and contributes nothing to the present analysis. The lower right-hand corner of the (1 017 ) pattern has also been masked out due to a transition from reflection geometry to thick-crystal transmission geometry.

The polaronic orbital polarization far above $T_{C}$ is unexpected and interesting in itself. Even more surprising, perhaps, is the observation of a dramatically different orbitalpolarization state at $120 \mathrm{~K}$, just a few degrees above $T_{C}$ $\sim 112 \mathrm{~K}$. Figure 3 shows a $120-\mathrm{K}$ diffuse scattering distribution around the $(200)$ Bragg reflection that looks very different from the $300-\mathrm{K}$ pattern of Fig. 2(a). The local displacements obtained from the fit to the $120-\mathrm{K}$ data are $u_{\mathrm{O} 1, x}=0.081(7) \AA$ and $u_{\mathrm{O} 2, y}=u_{\mathrm{O} 3, z}=-0.024(10) \AA$. These displacements reveal an octahedron that is now elongated along one of the in-plane directions and slightly compressed along the other two axes, indicating that the polaronic $e_{g}$ electrons now occupy $d\left(3 x^{2}-r^{2}\right)$ or $d\left(3 y^{2}-r^{2}\right)$ type orbit-
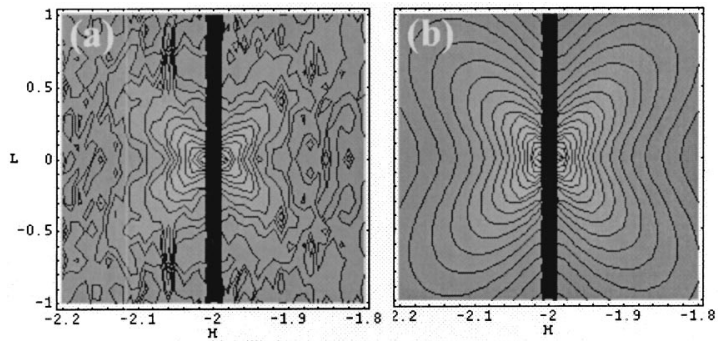

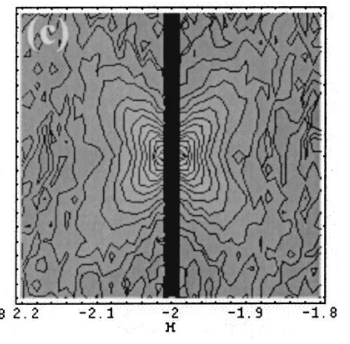

FIG. 3. Diffuse scattering data from the $(h 0 l)$ section surrounding the $(200)$ Bragg reflection at $120 \mathrm{~K}$. The format is identical to that of Fig. 2. 

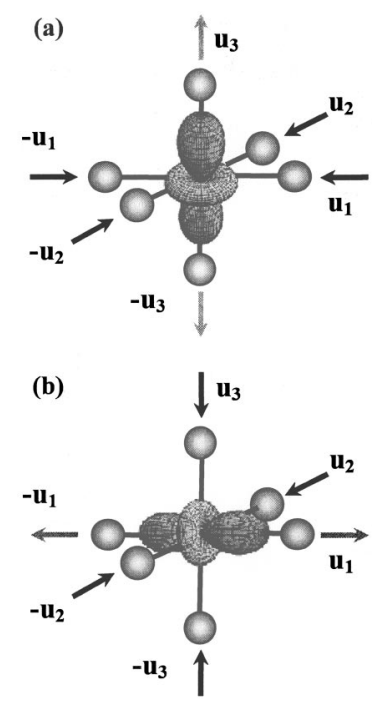

FIG. 4. Illustration of the correlated local displacements that comprise the Jahn-Teller distortions giving rise to the observed diffuse scattering patterns at (a) $300 \mathrm{~K}$ and (b) $120 \mathrm{~K}$. The occupied $e_{g}$ orbitals inferred from the displacement patterns are also shown.

als (Fig. 4). Thus an orbital polarization transition has occurred that preserves orbital symmetry but alters its orientation relative to the lattice. At room temperature, the occupied orbitals have their symmetry axes perpendicular to the perovskite layers (i.e., out of plane), whereas just above $T_{C}$, the orbital symmetry axis has shifted into the plane.

While the temperature dependence of the transition from out-of-plane to in-plane orbital polarization is not yet known, it occurs somewhere between 120 and $300 \mathrm{~K}$. Neutron powder diffraction data do reveal an anomalous but gradual increase in the in-plane $\mathrm{Mn}-\mathrm{O}$ bond distance within this temperature range, ${ }^{5,22}$ but other anomalies have been reported. Argyriou et al. have reported a transition from a polaron glass to a polaron fluid ${ }^{23}$ near room temperature, which may be related to the orbital polarization transition reported in the present work. Gordon et al. ${ }^{24}$ reported a distinct peak in the heat capacity of $\mathrm{La}_{1.2} \mathrm{Sr}_{1.8} \mathrm{Mn}_{2} \mathrm{O}_{7}$ near $250 \mathrm{~K}$ that could not be readily attributed to the lattice or magnetic degrees of freedom, and recent ellipsometry measurements indicate an anomaly in the optical conductivity just below room temperature associated with the orbital degrees of freedom. ${ }^{25}$ Furthermore, the adiabatic small polaron model has recently been reported to explain the high-temperature in-plane resistivity of $\mathrm{La}_{1.2} \mathrm{Sr}_{1.8} \mathrm{Mn}_{2} \mathrm{O}_{7}$, failing only below an intermediate temperature near $218 \mathrm{~K}^{26}$

The quantitative analysis of single-crystal $\mathrm{x}$-ray diffuse scattering data from $\mathrm{La}_{1.2} \mathrm{Sr}_{1.8} \mathrm{Mn}_{2} \mathrm{O}_{7}$ yields a $3 \mathrm{D}$ structural model of local JT distortions, and reveals the polaronic $e_{g}$ electrons to be orbitally polarized in the PI state above $T_{C}$. A transition from out-of-plane orbital to in-plane orbital character between 300 and $120 \mathrm{~K}$ should result in stronger interpolaron interactions (via stress-dipole coupling and/or enhanced orbital overlap) within the perovskite sheets, which act on the high concentration of polaronic charge carriers to facilitate the formation of quasistatic polaron correlations. Both of the experimentally observed polaron-correlation motifs, transverse (i.e., zig zag or CE type) $)^{11,12}$ and longitudinal (i.e., $L$ type), ${ }^{14}$ involve the cooperative occupation of inplane orbitals. Because the transfer of $e_{g}$ electrons to inplane orbitals reduces the exchange coupling between adjacent perovskite sheets along the out-of-plane direction, it restricts the effective dimensionality of the $e_{g}$ conduction band to be more purely two-dimensional. As such, polaronic orbital polarization effects in the bilayered manganites are both interesting and important to the origin of the CMR effect.

The authors thank Z. Islam for helpful discussions. This work was supported by the U.S. DOE, Office of Science, under Contract No. W-31-109-ENG-38. Work at NIST was supported in part by the Binational Science Foundation, Grant No. 2000073. S.R. acknowledges support from the Swiss NSF.
*Present address: Department of Physics and Astronomy, Brigham Young University, Provo, UT 84602.

${ }^{1}$ B. J. Campbell et al., in From Semiconductors to Proteins: Beyond the Average Structure, edited by S. J. L. Billinge and M. F. Thorpe (Kluwer Academic, Dordrecht/Plenum, New York, 2001).

${ }^{2}$ L. Vasilu-Doloc et al., Phys. Rev. Lett. 83, 4393 (1999).

${ }^{3}$ S. J. L. Billinge et al., Phys. Rev. Lett. 77, 715 (1996).

${ }^{4}$ C. H. Booth et al., Phys. Rev. Lett. 80, 853 (1998).

${ }^{5}$ M. Medarde et al., Phys. Rev. Lett. 83, 1223 (1999).

${ }^{6}$ P. G. Radaelli et al., Phys. Rev. B 55, 3015 (1997).

${ }^{7}$ T. Kimura et al., Phys. Rev. B 58, 11081 (1998).

${ }^{8}$ M. Kubota et al., J. Phys. Soc. Jpn. 68, 2202 (1999).

${ }^{9}$ D. N. Argyriou et al., Phys. Rev. B 61, 15269 (2000).

${ }^{10}$ S. Shimomura et al., Phys. Rev. Lett. 83, 4389 (1999).

${ }^{11}$ C. P. Adams et al., Phys. Rev. Lett. 85, 3954 (2000).

${ }^{12}$ P.-C. Dai et al., Phys. Rev. Lett. 85, 2553 (2000).

${ }^{13}$ M. Kubota et al., J. Phys. Soc. Jpn. 69, 1986 (2000).
${ }^{14}$ B. J. Campbell et al., Phys. Rev. B 65, 014427 (2001).

${ }^{15}$ K. Huang, Proc. R. Soc. London, Ser. A 190, 102 (1947).

${ }^{16}$ M. A. Krivoglaz, X-Ray and Neutron Diffraction in Non-ideal Crystals (Springer-Verlag, Berlin, 1996), english translation.

${ }^{17}$ B. J. Campbell, S. K. Sinha, R. Osborn, D. N. Argyriou, S. Rosenkranz, J. F. Mitchell, and J. W. Lynn (unpublished).

${ }^{18}$ H. Kanzaki, J. Phys. Chem. Solids 2, 24 (1957).

${ }^{19}$ M. Takata et al., J. Phys. Soc. Jpn. 68, 2190 (1999).

${ }^{20}$ T. Kimura et al., Phys. Rev. Lett. 81, 5920 (1998).

${ }^{21}$ A. Koizumi et al., Phys. Rev. Lett. 86, 5589 (2001).

${ }^{22}$ D. N. Argyriou et al., Phys. Rev. B 60, 6200 (1999).

${ }^{23}$ D. N. Argyriou et al., Phys. Rev. Lett. 89, 036401 (2002).

${ }^{24}$ J. E. Gordon et al., Phys. Rev. B 60, 6258 (1999).

${ }^{25}$ J. Kunze, S. Naler, J. Bäckström, M. Rübhausen, and J. F. Mitchell, Phys. Rev. Lett. (to be published).

${ }^{26}$ X. J. Chen, C. L. Zhang, C. C. Almasan, J. S. Gardener, and J. L. Sarrao (unpublished). 